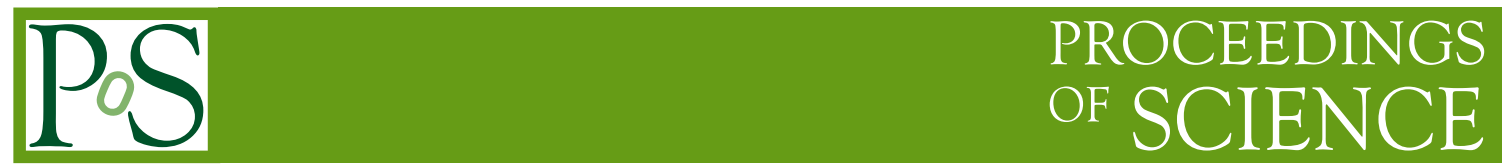

\title{
Top and heavy quark studies at linear colliders
}

\author{
Roman Pöschl ${ }^{a, *}$ \\ ${ }^{a}$ Université Paris-Saclay, CNRS/IN2P3, IJCLab, 91405 Orsay, France \\ On behalf of the ILC International Development Team Working Group 3 \\ E-mail: roman.poesch1@ijclab.in2p3.fr
}

The t quark has not yet been studied in the extremely favorable and low background environment of $\mathrm{e}^{+} \mathrm{e}^{-}$annihilation. This talk will review the opportunities for precision measurements of the $\mathrm{t}$ quark properties at the International Linear Collider (ILC). These include the archival measurement of the $t$ quark mass and the search for beyond-Standard Model contributions to the t quark electroweak form factors. The results are extended to the $b$ quark and the motivation for studies of lighter fermions are given.

*** The European Physical Society Conference on High Energy Physics (EPS-HEP2021), *** *** 26-30 July $2021 * * *$

*** Online conference, jointly organized by Universität Hamburg and the research center DESY ***

\footnotetext{
${ }^{*}$ Speaker, R.P. thanks the Conference Organisers, the ILC IDT and the ILC Software Working Group.
} 


\section{Introduction}

It is consensus in the community that the next project in high-energy particle physics should be a Higgs factory. There exist proposals for two linear colliders ILC and CLIC and two circular machines, FCCee and CEPC. The study of the process $\mathrm{e}^{+} \mathrm{e}^{-} \rightarrow \mathrm{f} \overline{\mathrm{f}}$ in high-energy $\mathrm{e}^{+} \mathrm{e}^{-}$collisions at Higgs factories is complementary to the actual Higgs program and bears discovery potential by precision measurements on its own. Linear colliders with their extendability in energy allow for detecting the onset of deviations in the electroweak couplings at smaller energies and for following the growth of effects towards higher energies. On the other hand the $t$ quark mass is an important parameter in global analyses of electroweak parameters [1]. If the scale of new physics would be comparable to the Planck scale then the actual t quark mass drives the stability of the vacuum.

\section{Determiniation of the top quark mass}

At the tit production threshold the small size of the quasi-bound state of the $\mathrm{t}^{\mathrm{t}}$ pair is an ideal premise for precision measurements. The cross section around the threshold is affected by several properties of the $\mathrm{t}$ quark and by QCD. These are the mainly top quark mass, the t quark width and the strong coupling constant $\alpha_{s}$. The dependence of the top-Yukawa coupling is rather weak. The parameters are extracted from a scan over the $t \bar{t}$ threshold. Typically 10 data points with around $10 \mathrm{fb}^{-1}$ at each point are needed for a precision of the top quark mass of about $50 \mathrm{MeV}$. The study in Ref. [2] searched for an optimal distribution of the data points around the threshold. It makes use of a non-dominated sorted genetic algorithm. In short, several sets of scanning points "breed" a new set of scanning points until the result, i.e. the physics parameters, cannot be further optimised. The left part of Fig. 1 shows the distribution of scanning points optimised for the determination of the $t$ quark mass and the $t$ quark width. The right part of the figure shows that an optimisation of threshold scan yields $25 \%$ improvement in statistical precision of $t$ quark mass compared with scan using equally distributed scan points.

Initial state radiation, i.e. the process $\mathrm{e}^{+} \mathrm{e}^{-} \rightarrow \mathrm{t} \overline{\mathrm{t}}+\gamma$ allows to determine the $\mathrm{t}$ quark mass at different (squared) centre-of-mass energies $\mathrm{s}^{\prime}=\mathrm{s}\left(1-2 \mathrm{E}_{\gamma} / \sqrt{\mathrm{s}}\right)$ and therefore gives evidence for the running of the $t$ quark mass as expected from renormalisation in a consistent field theoretical treatment. A conceptual study has been published in Ref. [3]. The left hand side of Fig. 2 shows pseudo data generated for the $\mathrm{e}^{+} \mathrm{e}^{-} \rightarrow \mathrm{t} \overline{\mathrm{t}}+\gamma$ cross section for a centre-of-mass energy of $500 \mathrm{GeV}$.

The renormalisation scale is chosen to be the momentum of the top quark, i.e. $\mathrm{R}=\mathrm{m}_{\mathrm{t}}^{1 \mathrm{~S}} \mathrm{v}^{*}$, with $\mathrm{m}_{\mathrm{t}}^{1 \mathrm{~S}}$ being the $\mathrm{t}$ quark mass at the $1 \mathrm{~S}$ resonance peak and with $\mathrm{v}^{*}$ being the velocity of the $\mathrm{t}$ quark in the $t \bar{t}$ centre-of-mass frame. Results have been obtained for a fixed point close to the $t \bar{t}$ threshold and three further bins in R. The running of the t quark mass (MSR mass) is evident from the right hand panel of Fig. 2. Though for the last three bins the error is comparatively large, the running of the $t$ quark mass is confirmed with a significance of more than $5 \sigma$.

\section{Electroweak couplings of the top quark and lighter quarks}

In general electroweak di-fermion production in the process $\mathrm{e}^{+} \mathrm{e}^{-} \rightarrow \mathrm{f} \overline{\mathrm{f}}$ is driven by the electromagnetic charges $\mathrm{Q}_{\mathrm{e}, \mathrm{f}}^{\gamma}$ of the initial and final state particles to the photon and by the couplings 

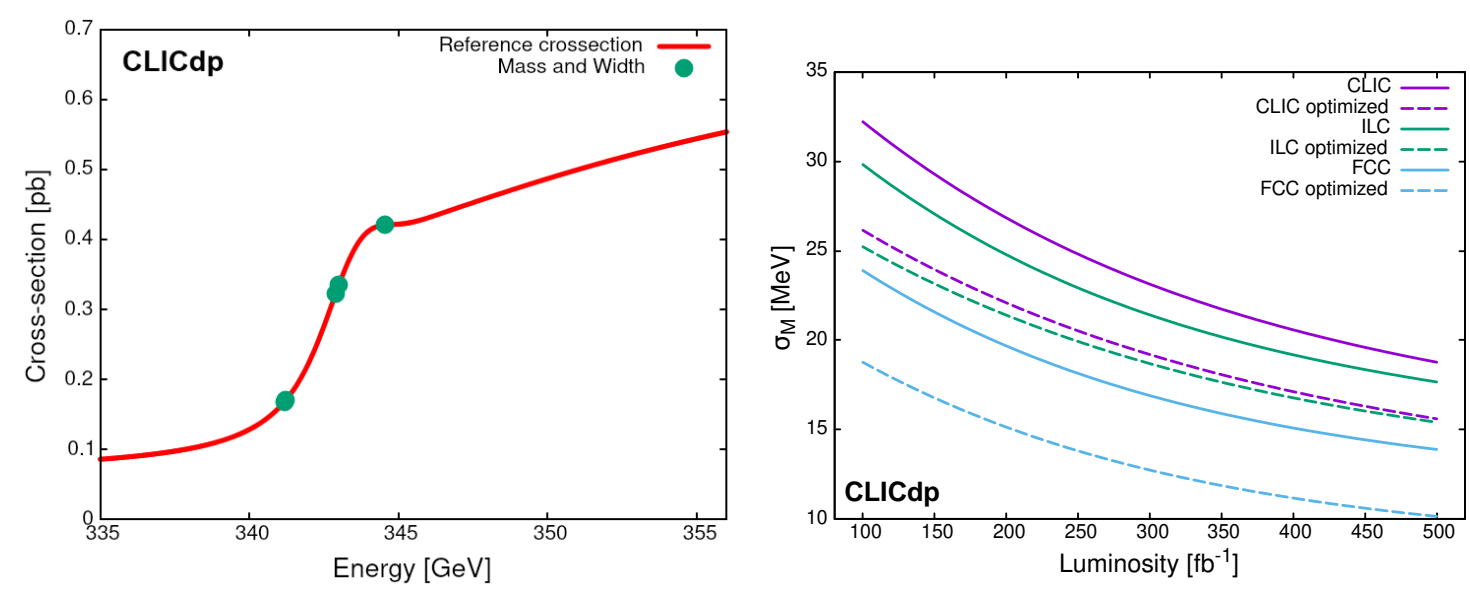

Figure 1: Left: Distribution of scanning points in a threshold scan for optimising the measurements of mass and the width of the t quark. Right: Improvement of the statistical precision on the determination of the $t$ quark mass achieved with the optimised scenario for different Higgs factories.
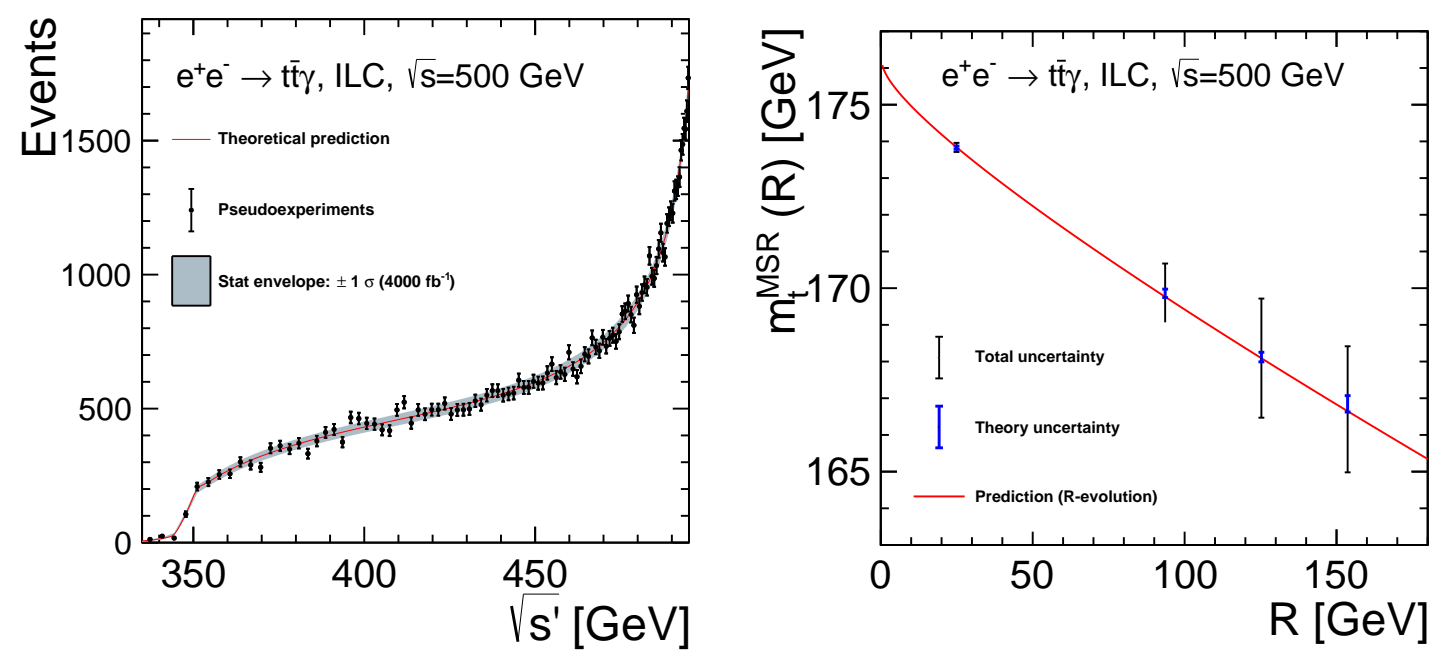

Figure 2: Left: Pseudo data of the process $e^{+} e^{-} \rightarrow t \bar{t}+\gamma$ at $\sqrt{s}=500 \mathrm{GeV}$ using the ILC luminosity spectrum.Right: Expected running of the MSR mass as a function of the scale $R$.

$\mathrm{g}_{(\mathrm{e}, \mathrm{f})_{\mathrm{L}}}^{\mathrm{Z}}, \mathrm{g}_{(\mathrm{e}, \mathrm{f})_{\mathrm{R}}}^{\mathrm{Z}\left({ }^{\prime}\right)}$ of fermions with left-handed and right-handed helicities to the $\mathrm{Z}$ boson and to a potential $Z^{\prime}$ boson of new physics. In a general form the couplings can be expressed as:

$$
Q_{e_{i} f_{j}}=Q_{e}^{\gamma} Q_{f}^{\gamma}+\frac{g_{e_{i}}^{Z} g_{f_{j}}^{Z}}{\sin ^{2} \theta_{W} \cos ^{2} \theta_{W}} \frac{s}{s-m_{Z}^{2}+i \Gamma_{Z} m_{Z}}+\sum \frac{g_{e_{i}}^{Z^{\prime}} g_{f_{j}}^{Z^{\prime}}}{\sin ^{2} \theta_{W} \cos ^{2} \theta_{W}} \frac{s}{s-m_{Z^{\prime}}^{2}+i \Gamma_{Z^{\prime}} m_{Z^{\prime}}}
$$

with $i, j=L, R$ and $\theta_{\mathrm{W}}$ being the weak mixing angle at Born level. The first part of the equation describes the electromagnetic couplings to the photon. The second part describes the couplings of the fermions to the $\mathrm{Z}$ boson. This second term may be affected by $\mathrm{Z}-\mathrm{Z}^{\prime}$ mixing as for example 

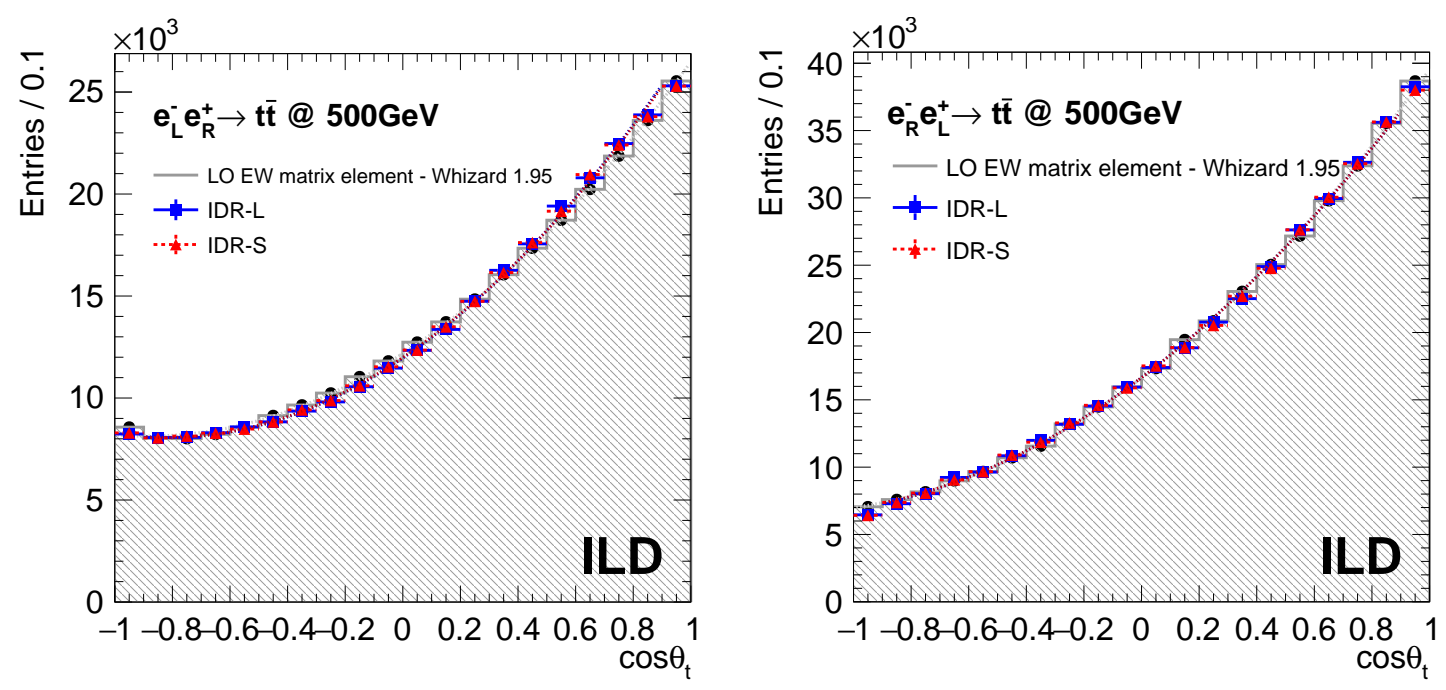

Figure 3: Polar angle distributions of the top quark for different configurations of the beam polarisation. Note for completeness that IDR-L and IDR-S represent different variants of the ILD Detector Concept.

suggested in [4]. The third term takes into account couplings to new vector bosons $Z^{\prime}$. The relative importance of the contributions is determined by the Breit-Wigner functions characterised by the squared centre-of-mass energy s and the mass $m_{Z}\left(m_{Z^{\prime}}\right)$ and the width $\Gamma_{Z}\left(\Gamma_{Z^{\prime}}\right)$ of the $Z\left(Z^{\prime}\right)$ boson.

The differential cross-sections for s-channel, i.e. $f \neq e$, di-fermion production using fully polarised electron and positron beams read:

$$
\begin{aligned}
& \frac{d \sigma}{d \cos \theta}\left(e_{L}^{-} e_{R}^{+} \rightarrow f \bar{f}\right)=\Sigma_{L L}(1+\cos \theta)^{2}+\Sigma_{L R}(1-\cos \theta)^{2}+\left(\Sigma_{L, L R}^{\prime} / \gamma_{f}\right) \sin ^{2} \theta \\
& \frac{d \sigma}{d \cos \theta}\left(e_{R}^{-} e_{L}^{+} \rightarrow f \bar{f}\right)=\Sigma_{R L}(1-\cos \theta)^{2}+\Sigma_{R R}(1+\cos \theta)^{2}+\left(\Sigma_{R, R L}^{\prime} / \gamma_{f}\right) \sin ^{2} \theta
\end{aligned}
$$

Here, $\theta$ is the polar scattering angle and $\gamma_{f}$ the Lorentz factor of the final state fermion. The terms containing $(1 \pm \cos \theta)^{2}$ or $\sin ^{2} \theta$ represent helicity conserving or violating contributions at the final state vertex. In the Standard Model the helicity violating contributions vanish at relativistic energies. The coefficients $\Sigma$ and $\Sigma^{\prime}$ are functions of the couplings defined in Eq. 1. In case of e.g. the $\mathrm{Z}$ boson these couplings appear as vector couplings $\propto\left(\mathrm{g}_{\mathrm{f}_{\mathrm{L}}}^{\mathrm{L}}+\mathrm{g}_{\mathrm{f}_{\mathrm{R}}}^{\mathrm{Z}}\right)$ or as parity-violating axial-vector couplings $\propto \beta_{\mathrm{f}}\left(\mathrm{g}_{\mathrm{f}_{\mathrm{L}}}^{\mathrm{Z}}-\mathrm{g}_{\mathrm{f}_{\mathrm{R}}}^{\mathrm{Z}}\right)$ with $\beta_{\mathrm{f}}$ being the velocity of the final state fermion. The difference in the coefficients leads to the well known left-right and forward-backward asymmetries.

Figure 3 shows the polar angle distribution of the top quarks in the process $\mathrm{e}^{+} \mathrm{e}^{-} \rightarrow \mathrm{tt}$ at $\sqrt{s}=500 \mathrm{GeV}$ [5]. The result has been obtained using full simulation of the ILD detector [6] and assuming $3200 \mathrm{fb}^{-1}$ integrated luminosity shared equally between two different configurations of the beam polarisation. The generated spectra are accurately reproduced. The different shapes of the distributions are reminiscent of the different weights with which left and righthanded couplings of the $t$ quark enter the distrubtions. Characteristic observables as the crosssections and the forward-backward asymmetries can be measured with a statistical accuracy of $0.2-0.3 \%$ and $0.5-0.7 \%$, respectively. This sets the goal for systematic and theoretical uncertainties. 


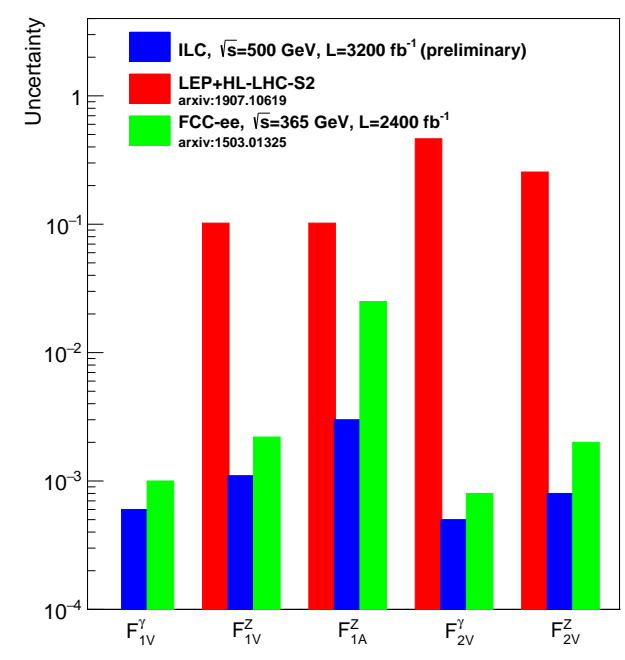

Figure 4: Precisions the on electromagnetic t quark form factors expected after ILC compared with those expected after the full HL-LHC running and an estimation for FCC-ee after $2400 \mathrm{fb}^{-1}$.

The precisions on the cross-sections and the forward-backward asymmetries are translated into precisions on electromagnetic form factors of the $\mathrm{t}$ quark. Figure 4 shows the precisions at the $1 \sigma$ level expected at the ILC. The precisions are compared with those expected after the full HLLHC running and estimations produced for FCCee [7] at the same confidence level. For the ILC, the two sets $F_{1}$ and $F_{2}$ have been extracted separately but within each set the uncertainties have been extracted simultaneously. The projections for HL-LHC are derived from the individual constraints of EFT Wilson coefficients presented in Table C2.3 of Ref. [8]. The figure demonstrates clearly the superiority of a $\mathrm{e}^{+} \mathrm{e}^{-}$collider compared with HL-LHC. For about equal total integrated luminosities the results are similar for circular and linear colliders. The exception is the axial-vector form factor $\mathrm{F}_{1 \mathrm{~A}}^{\mathrm{Z}}=\frac{1}{2} \beta_{\mathrm{f}}\left(\mathrm{g}_{\mathrm{t}_{\mathrm{L}}}^{\mathrm{Z}}-\mathrm{g}_{\mathrm{t}_{\mathrm{R}}}^{\mathrm{Z}}\right)$. This form factor benefits from the higher velocity of the $t$ quark at higher centre-of-mass energies available at linear colliders.

The $\mathrm{b}$ quark is the second heaviest fermion and builds, in the Standard Model, an iso-doublet with the t quark. Be reminded on the discrepancy between LEP and SLC on the weak mixing angle and that at LEP the parameter has been extracted from the forward-backward asymmetry observed for the $b$ quark [9]. This motivates further studies at future $\mathrm{e}^{+} \mathrm{e}^{-}$colliders. Figure 5 shows the polar angle distributions for the process $\mathrm{e}^{+} \mathrm{e}^{-} \rightarrow \mathrm{b} \overline{\mathrm{b}}$ at a centre-of-mass energy of $250 \mathrm{GeV}$ for an integrated luminosity of $2000 \mathrm{fb}^{-1}$ and two different configurations of the beam polarisation.

The generated distributions are accurately reproduced for a wide range of the polar angle. The difference in the shape is even more striking than in case of the top quark. In particular the configuration $\mathrm{e}_{\mathrm{R}}^{-} \mathrm{e}_{\mathrm{L}}^{+}$gives a good access to right-handed polarised $\mathrm{b}$ quarks. Figure 6 gives the expected precision in terms of a) the coefficients of the helicity amplitudes defined in Eq. 2 and b) in terms of the left and right-handed couplings that are expected from a running of the ILC at the Z-Pole $[10,11]$. For the former case there doesn't exist a result to compare with. For the latter the improvement w.r.t. LEP/SLC is obvious.

The results introduced before are subject to a global analysis in the EFT framework as shown in Fig. 7 [8]. From this three conclusions can be drawn: a) Translation of results into EFT language confirms the superiority of $\mathrm{e}^{+} \mathrm{e}^{-}$colliders w.r.t. HL-LHC; $b$ ) Several operators benefit already from running at $250 \mathrm{GeV}$; c) Top specific operators are constrained by running at $500 \mathrm{GeV}$.

Reference [14] points out that the sensitivity to four-fermion operators improves considerably with higher energies. On the other hand Ref. [15] underlines for models based on Grand Higgs Unification a) that deviations from the Standard Model are already measurable at $\sqrt{s}=250 \mathrm{GeV}$, 

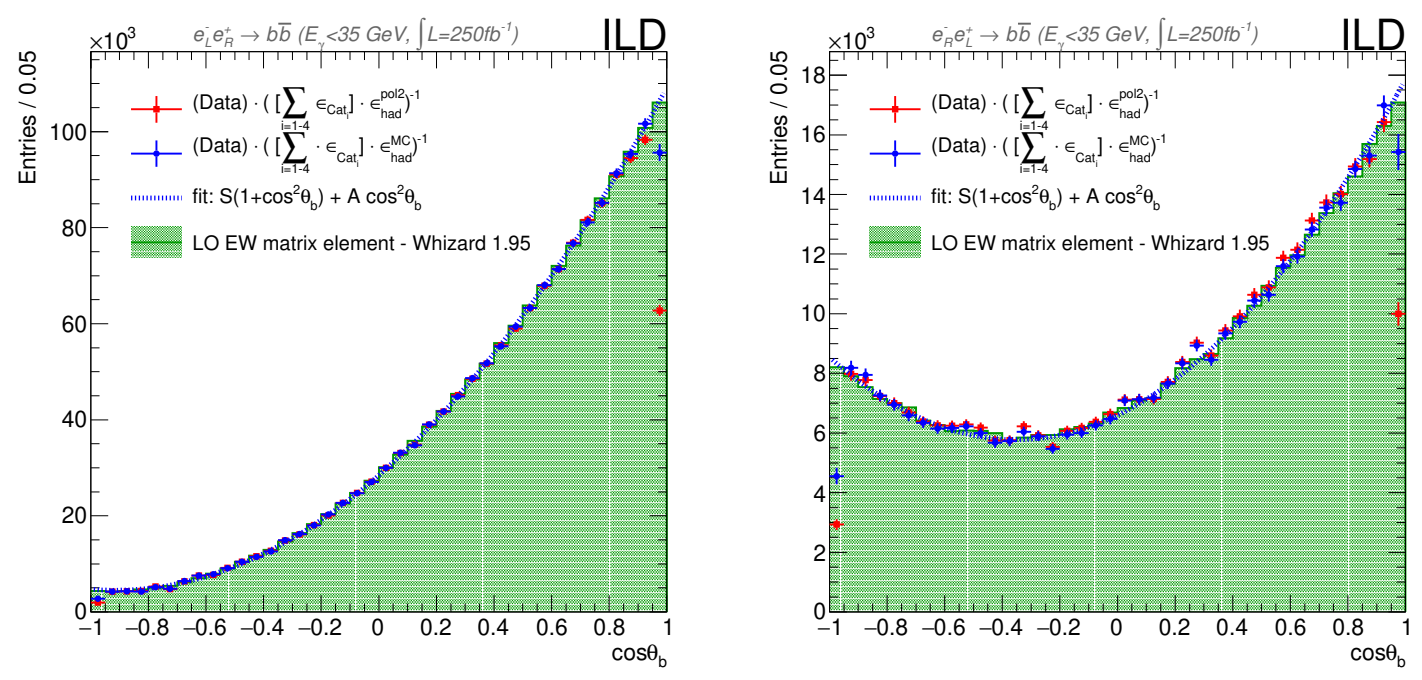

Figure 5: $\cos \theta_{b}$ distributions obtained for $e_{L}^{-} e_{R}^{+}$(left) and $e_{R}^{-} e_{L}^{+}$(right). The generator distribution is the green histogram and the red and blue dots show the reconstructed distributions after correction charge for charge migration and two different methods for the correction for efficiency and acceptance.
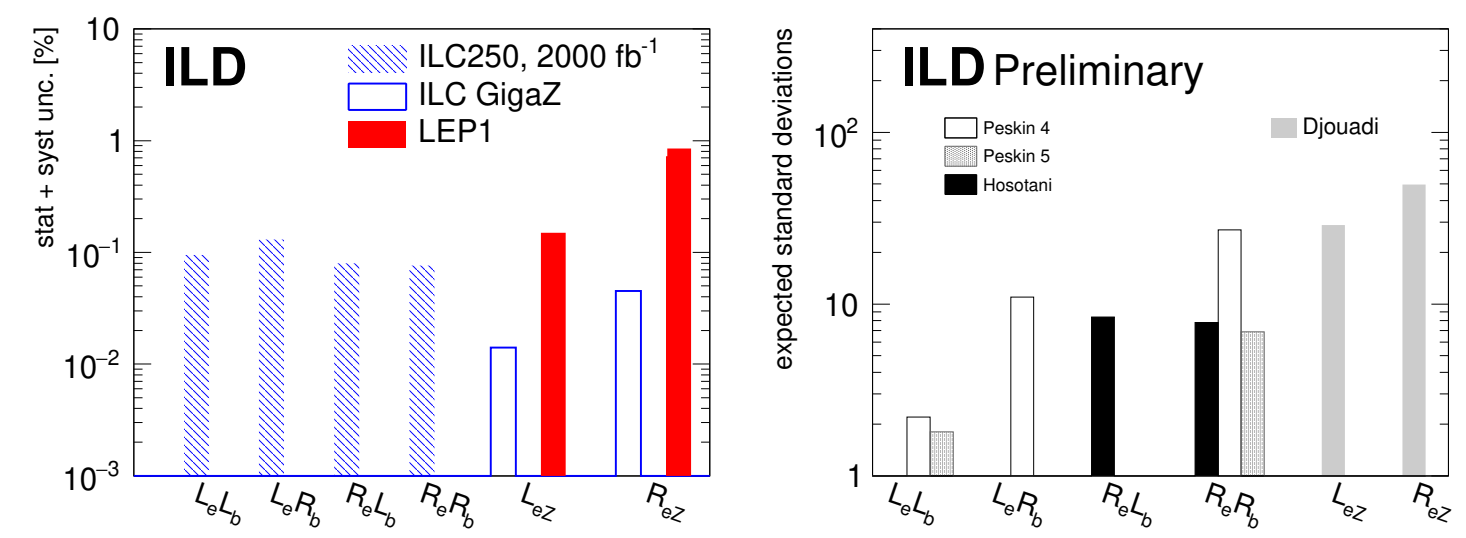

Figure 6: Left: Precisions expected on the coefficients of the helicity ampliudes defined in Eq. 2 (here expressed as $I_{e} J_{b}$ with $I, J=L, R$ ) and the left and right-handed couplings of the q quark to the $Z$ as expected from a running of the ILC on tne Z-Pole. Right: Deviations from the SM predictions in $g_{b_{j}}^{Z}, g_{b_{j}}^{Z}$ and the helicity amplitudes $Q_{e_{i} b_{j}}$, in standard deviations, from combined ILC250/Z-Pole running, expected from new physics models with Randall-Sundrum extra dimensions [4, 12, 13]. 


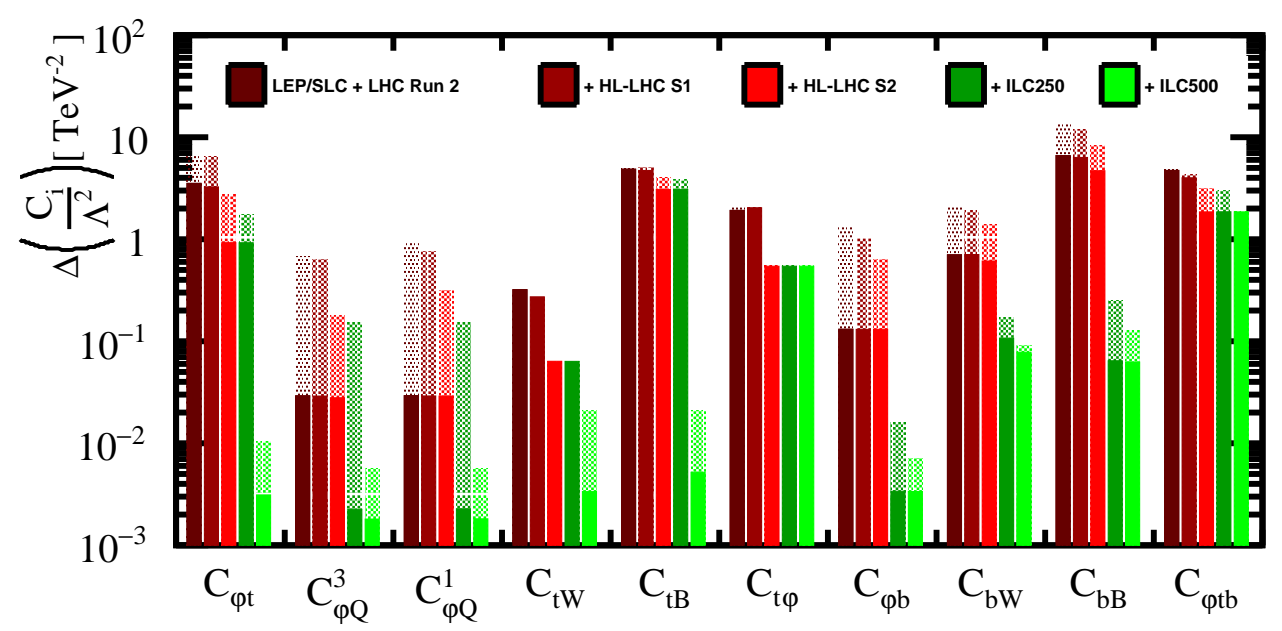

Figure 7: Prospects on the expected precisions of the Wilson Cofficients of EFT operators relevant for the $e^{+} e^{-} \rightarrow t \bar{t}$ and $e^{+} e^{-} \rightarrow b \bar{b}$ processes for HL-LHC and future $e^{+} e^{-}$colliders. The solid lines are the individual constraints and the shaded bars are the constraints resulting from a 10-parameter fit.

b) that the effects get amplified by polarisation, c) that these effects are further amplified at higher energies (when the effects of the new propagators get more important) and d) that effects are visible also for quarks lighter than $t$ or $b$.

\section{Summary and outlook}

This article gives a brief overview on the status of the determination of the top quark mass and the measurement of electroweak couplings of quarks as can be expected from experiments at linear $\mathrm{e}^{+} \mathrm{e}^{-}$colliders. The determination of the top quark mass may benefit from an optimisation of the points along a threshold scan. The optimisation made use of a genetic sorting algorithm and the statistical precision can be improved by around $25 \%$ giving more confidence that a precision of the top quark mass of $50 \mathrm{MeV}$ is in reach. Radiative events allow for measuring with great evidence the running of the top quark mass. This has been shown at the example of a generator study that imply efficiencies expected in a real experiment. Linear Colliders allow for a powerful program to determine the electroweak couplings of fermions. Beam polarisation and extendability in energy yields for precision measurement of left and right handed electroweak couplings. If present the onset of new physics can already be observed at small centre-of-mass energy and amplified effects can be followed up to higher energies. The variety of models of new physics motivates extending the study to all fermion types. An analysis for $\mathrm{e}^{+} \mathrm{e}^{-} \rightarrow \mathrm{c} \overline{\mathrm{c}}$ has been published in [16] and a study of $\mathrm{e}^{+} \mathrm{e}^{-} \rightarrow \mathrm{s} \overline{\mathrm{s}}$ has just been started. 


\section{References}

[1] M. Reece, "Physics at a Higgs Factory", Int. J. Mod. Phys. A 31 (2016) no. 33, 1644003, arXiv: 1609.03018 [hep-ph].

[2] K. Nowak and A. F. Zarnecki, "Optimising top-quark threshold scan at CLIC using genetic algorithm”, JHEP 07 (2021) 070, CLICdp-Pub-2021-002, arXiv: 2103.00522 [hep-ex].

[3] M. Boronat, E. Fullana, J. Fuster, P. Gomis, A. Hoang, V. Mateu, M. Vos, and A. Widl, "Top quark mass measurement in radiative events at electron-positron colliders", Phys. Lett. B 804 (2020) 135353, CLICdp-PUB-2019-005, IFIC-19-036, IFT-UAM/CSIC-19-122, UWThPh 2019-26, UWThPh 2019-26, arXiv: 1912.01275 [hep-ph].

[4] A. Djouadi, G. Moreau, and F. Richard, "Resolving the A(FB)**b puzzle in an extra dimensional model with an extended gauge structure", Nucl.Phys. B773 (2007) 43-64, LPT-ORSAY-06-060, LAL-ORSAY-06-144, arXiv:hep-ph/0610173 [hep-ph].

[5] M. Amjad, A. Irles, V. Lohezic, Y. Okugawa, R. Pöschl, F. Richard, H. Yamamoto, and R. Yonamine, "Bottom and top reconstruction as benchmarks for ILD", ILD-PHYS-PUB-2019-007. https: //confluence. desy . de/display/ILD/ILD+ notes?preview=/42357928/165987677/ILD-PHYS-PUB-2019-007 . pdf.

[6] ILD Concept Group Collaboration, H. Abramowicz et al., "International Large Detector: Interim Design Report”, DESY-20-034, KEK 2019-57, arXiv : 2003.01116 [physics.ins-det].

[7] P. Janot, "Top-quark electroweak couplings at the FCC-ee", JHEP 04 (2015) 182, arXiv: 1503.01325 [hep-ph].

[8] G. Durieux, A. Irles, V. Miralles, A. Peñuelas, R. Pöschl, M. Perelló, and M. Vos, "The electro-weak couplings of the top and bottom quarks - global fit and future prospects", IFIC/19-33, FIC/19-33, FTUV/19-0724, arXiv: 1907.10619 [hep-ph]. [JHEP12,098(2019)].

[9] ALEPH, DELPHI, L3, OPAL, SLD, LEP Electroweak Working Group, SLD Electroweak Group, SLD Heavy Flavour Group Collaboration, S. Schael et al., "Precision electroweak measurements on the $Z$ resonance", Phys. Rept. 427 (2006) 257-454, SLAC-R-774, arXiv: hep-ex/0509008.

[10] LCC Physics Working Group Collaboration, K. Fujii et al., "Tests of the Standard Model at the International Linear Collider", DESY 19-146, KEK Preprint 2019-22, SLAC-PUB-17467, DESY-19-146, arXiv: 1908.11299 [hep-ex].

[11] A. Irles, R. Pöschl, F. Richard, and H. Yamamoto, "Complementarity between ILC250 and ILC-GigaZ”, in Linear Collider Community Meeting. 5, 2019. arXiv: 1905.00220 [hep-ex]. 
[12] S. Funatsu, H. Hatanaka, Y. Hosotani, and Y. Orikasa, "Distinct signals of the gauge-Higgs unification in $e^{+} e^{-}$collider experiments", Phys. Lett. B775 (2017) 297-302, KEK-TH-1978, OU-HET-934, arXiv: 1705.05282 [hep-ph].

[13] J. Yoon and M. E. Peskin, "Fermion Pair Production in SO(5) x U(1) Gauge-Higgs Unification Models", SLAC-PUB-17356, arXiv: 1811.07877 [hep-ph].

[14] G. Durieux, M. Perelló, M. Vos, and C. Zhang, "Global and optimal probes for the top-quark effective field theory at future lepton colliders", JHEP 10 (2018) 168, DESY-18-096, IFIC-18-27, DESY 18-096, IFIC 18-27, arXiv: 1807.02121 [hep-ph].

[15] S. Funatsu, H. Hatanaka, Y. Hosotani, Y. Orikasa, and N. Yamatsu, "Fermion pair production at $e^{-} e^{+}$linear collider experiments in GUT inspired gauge-Higgs unification", Phys. Rev. D 102 (2020) no. 1, 015029, OU-HET-1060, KYUSHU-HET-214, arXiv : 2006.02157 [hep-ph].

[16] ILD Collaboration, A. Irles, R. Pöschl, and F. Richard, "Production and measurement of $e^{+} e^{-} \rightarrow c \bar{c}$ signatures at the $250 \mathrm{GeV}$ ILC", in International Workshop on Future Linear Colliders. 2, 2020. arXiv:2002.05805 [hep-ex]. 